\title{
Embedded Home Automation for Visually Impaired
}

\author{
V. Ramya \\ Assistant Professor \\ Department of Computer Science and \\ Engineering,AnnamalaiUniversity, Chidambaram, \\ Tamilnadu
}

\author{
B. Palaniappan \\ Dean, FEAT, Head \\ Department of Computer Science and Engineering, \\ AnnamalaiUniversity, Chidambaram, Tamilnadu
}

\begin{abstract}
This paper aims to develop an embedded system which is used to control the home appliances through voice and mobile for the visually impaired people and also for the physically challenged people. Though various techniques have been proposed and are available currently, most of them are not user friendly for the visually impaired people and thus a friendly system has been developed for them. As speech is the preferred mode of operation for these people, this project intends to make use of voice oriented command words for controlling purposes. Further the hand-held unit has a microphone to receive the voice input and is a wireless module which uses the Radio Frequency (RF) technology. This project uses the DTMF technique in the mobile device so as to avoid any scenario involving visual interaction for the benefit of the user. Also in this module there is a voice feedback as an acknowledgement both for the voice controlled automation and automation through mobile.
\end{abstract}

\section{Keywords}

DTMF Technology, Embedded System, Home Automation, PIC Microcontroller, RF technology, Voice feedback.

\section{INTRODUCTION}

The world is moving as more and more process are being automated. Basically there are two main reasons for automating a process. First, humans tend to get bored repeating a process again and again. Second, as human beings they are prone to making errors. Automation solves both the problems. Home Automation is still a relatively new concept in India and it has reached the urban high society people, but yet to penetrate into the rural and middle class society. Contrary to popular benefits home automation systems are very costly, by spending a small but reasonable fraction of the total cost of a newly constructed home; the home can be fully automated for the convenience of the user. In a world where electronic gadgets and gizmos are the order of the day and have made life easy, home automation has become a necessity [1]. Though many popular home automation modules are available in the market, very few of them are compatible when we need to use them in a system for the physically challenged and also for the visually impaired people. The proposed system has two set of modules, control unit and the relay unit. Both the units are connected wirelessly with each having separate microprocessors so that work of each microprocessor is defined separately. Also in this module there is a voice feedback as an acknowledgement for both vice controlled automation and automation through mobile. This will be of great help for the visually impaired user to ascertain the status of the device on issue of the command signal or the command word.
Visually impaired people mainly rely on voice commands, voice menu or voice feedbacks for any control operation. Here we integrate voice features into home automation system. Home automation is achieved by the use of voice control and mobile in this project, voice-control is used, if in case the user is within the premises of the home where the appliances are to be controlled. Automation through mobile is exercised when the user is outside the premises. When voice control is used, the user should be able to select a particular device and should be able to disable it. This should be done with the help of a wireless handheld module which provides more flexibility than a wired control module. There should be a voice feedback acknowledging that the particular task has been accomplished as per the user's wish. When the mobile control is used, the user should be able to control remotely the specific devices and enable or disable them using the mobile. Since the devices are controlled remotely, a voice feedback must be given as an acknowledgement for the completion of the specific task. This will be of great help for the visually impaired user to ascertain the status of the device on issue of the command signal or command word.

\section{RELATED WORK}

Van Der Werff et al [1] proposed home server built upon an SMS/GPRS (Short Message Service/ General Packet Radio Service) mobile cell module and a microcontroller, allowing a user to control and monitor any variables related to the home by using any java capable cell phone. A java application is designed for the mobile, which enables the cell phone to send commands and receive alerts through the cell module. It is a mobile based automation system in which the home appliances are controlled by the home server, which operates according to the user commands received from the mobile phone via cellular modem.

Andreas Rosendahl et al [2]proposed a system in which the devices to be controlled are listed out and a hierarchy is formed. Since the hierarchy reflects a well-known structure, the navigation stays intuitive despite the limited interaction capabilities of the small device. It is a mobile home automation system, which merges the value added services with home automation technology. A user interaction application is designed for the mobile using J2ME. However the concrete presentation of possible commands depends on the J2ME implementation on the particular device. The module is designed to work with the lower bandwidth offered by GPRS (General Packet Radio Service), which is available in most areas covered by GSM (Global System for Mobile Communications) and offers the advantage of packet-oriented data communication.

BarisYukeskkaya et al [3] proposed a home automation system based on speech, Internet and GSM technologies. For speech controlled automation a speech recognition program is 
written to control the house by means of human voice. Dynamic Time Warping (DTW) algorithm is made use of for speech recognition. For the internet feature, a web server is built to take requests from remote clients. For the GSM part, if an action is chosen through the java application in the mobile, an appropriate feedback SMS will be sent back to the user as a confirmation. The clients can send requests to the home appliances. The home appliances can send their statuses to be displayed for the remote client through the server.

Renato Jorge CaleriaNunes [4] proposed a decentralized supervision system for home automation. For the supervision model he proposed a simple approach, based on the use of IF/ THEN clauses. These can be mapped, at a user level, on the intuitive notion of scenarios. This paper proposes a hierarchy of entities, where the home automation devices test Processes (TP) and Scenario processes (SP) are identified. Each TP is associated with a specific set of devices, receiving only their status information and evaluating the tests that refer to those devices. The SP is responsible for the effective execution of scenarios. For example, while a TP may receive 1000 messages from a single sensor, the SP is notified when a given test becomes true or false, having a small flow of incoming messages.

Ali ZiyaAlkar and UmitBuhaur [5] proposed an internet based wireless home automation system for multifunctional devices. They modelled the system with three different units namely, PC unit, Master node unit and the slave nodes units. The PC unit acts as the manger unit providing access to the database of the home automation system. The status changes that are reflected to the database are transferred to the slave nodes. The communication between the master and the slave node is established through RF. The modulation of the RF communication is performed using an FM modulator working at $433 \mathrm{MHz}$ frequency and $9600 \mathrm{Kbps}$ speed.

KwangYeol Lee and Jae Weon Choi [6] proposed a remote controlled home automation system using the Bluetooth home network. In this paper they use Bluetooth to connect the various devices to the server Bluetooth. The server Bluetooth is connected to the $\mathrm{PC}$ which can be accessed from anywhere through the internet. On initialization, the server Bluetooth sends DCP (Device Checking Packet) to each device Bluetooth. Device Bluetooth scans a server Bluetooth and sends a DDP (Device Detection Packet) to the server Bluetooth. The server Bluetooth sends an ACKP (Acknowledgement Packet) or NACK (NonAcknowledgment Packet).On device checking, server Bluetooth sends a DCP to device Bluetooth to check. If server Bluetooth does not receive an receive an acknowledgment from the device Bluetooth, the server Bluetooth assumes that the device Bluetooth is not connected or not working. On data transmission, the server Bluetooth sends a CP (Control Packet) to the device Bluetooth. If the device Bluetooth receives a control packet, the device Bluetooth sends an acknowledgement and updates a MSV (Measured Sensor Value). They are communicating between the server Bluetooth and the device Bluetooth repeatedly.

Rune Torbensen [8] proposed an open home automation system consisting of products from many diverse vendors. This paper proposed an open framework for integrating new products with existing home automation equipment consisting of two parts: An upgradable heterogeneous network infrastructure and a method for describing embedded devices. The infrastructure consists of scalable distributed gateway architecture that provides data communication between different types of network. Using generic communication modules, that encapsulate communication technology, the infrastructure and legacy products can be upgraded to take advantage of the latest data communication technologies. To enable services and allow semi-automatic system configuration, devices entering the framework must provide a device description language comprising a syntactic interface description part and a semantic description part. By this infrastructure setup natural language components vendors can now describe new innovative features that are not yet supported in any standard.

Zhi Li and Guanglic ZHANG [10] proposed a physical activities healthcare system based on wireless sensing technology. Bluetooth is used for wireless connectivity. This paper proposed a wearable wireless sensors unit which records physical activities and ambulatory electrocardiogram and all these data are transmitted to a hand-held device using Bluetooth. The hand-held unit monitors the entire exercise process and checks the heart rate. When there is any abnormality (Which is detected by the software running in the hand-held unit), the hand-held unit can access internet via Wfi for telemedicine functions.

\section{HOME AUTOMATION}

Normally home automation employs a catchy visual front end where the user needs to select the options to perform a specific action. This is normally done with the help of a PC. In some other methods hand-held units need to be operated upon for specific actions to be performed. Here too visuals act as the primary mode of interface with the user. Further in these cases there are no voice feedbacks for the user to confirm whether the intended action has been performed or not [3].This project tends to implement an embedded system for the visually impaired people, which enable them to control home appliances. Here a home automation system is implemented which can be controlled simultaneously through voice and also through mobile as shown in fig 1(a) \& 1(b). This is achieved by integrating the GSM mobile technology and the voice control mechanism with a microcontroller along with other additional necessary hardware circuitry. The home automation through voice control has been implemented with the help of HM2007, which is a voice recognition chip. Home automation through mobile has been implemented with the help of CM8870, which is a DTMF receiver. The hand held unit through which voice commands are spoken into is a wireless module, which uses the Radio Frequency technology.



Fig.1(a) Control Unit 


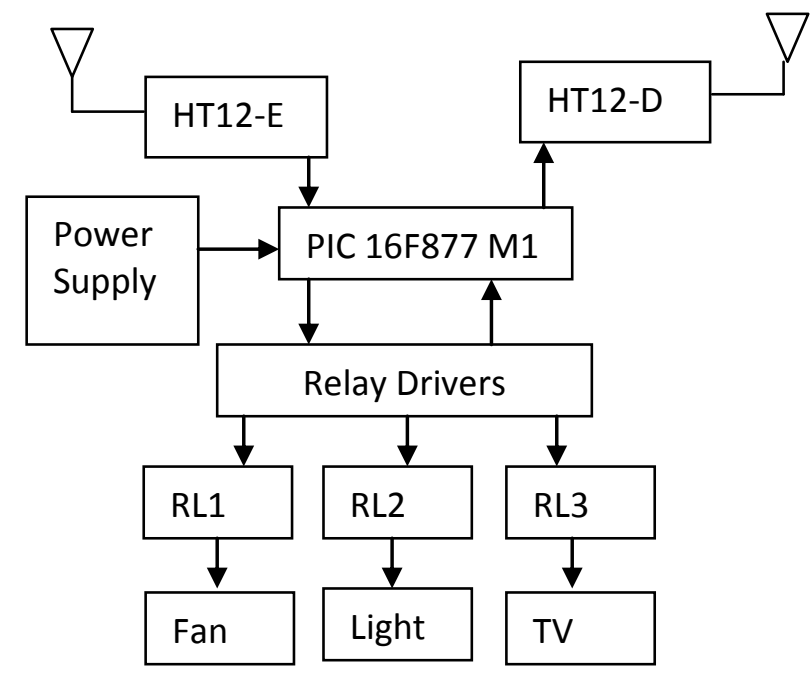

Fig.1 (b) Relay unit

\subsection{Automation through voice}

Voice command is the preferred mode of operation for the visually impaired and also for the physically challenged people. In voice controlled automation, the voice commands to control specific devices are first stored in a memory device. When the user intends to control a device, the user speaks the control word into the microphone, which acts as the input to the hardware circuitry [9]. The voice recognition chip (HM2007) gets the input command word and checks whether it is already stored in the memory. If the command word is already stored, then the command word is recognized and the voice is converted in to its corresponding pre-determined digital output. The microcontroller receives the output from the voice recognition chip, which is a digital control word and then acts according to the pore-described functionality for the specific input, which is to accurate a specific relay. The relay thus actuates the device attached to it, thereby fulfilling our intention to control a device. Voice feedbacks to indicate whether a specific task has been performed as instructed are already stored in a memory chip. So when the corresponding operation is performed, as per the user's wish, the specific voice feedback is given out through a speaker, which is an acknowledgement for the execution of the specific task to the user. For situations where the user gives commands, for actions which have already taken place, a unique voice message mentioning "Task already done" is played. Initially the microphone which is used to give voice command input is wired to the control module. To increase the flexibility of the model, wireless mikes are used hence, the hand held unit has a transmitter and receiver, which transmits the digital output of the HM2007 chip to the microcontroller attached to the relay circuitry. The microcontroller actuates the relays connected to it based on the digital input. The relay used here is a double pole throw relay. One set of contacts is used to actuate the device and the other set of the contacts is used to determine in which state the relay is hence the state of the device too is known. For example, when the user tries to switch OFF a device which is already switched OFF, the microcontroller ascertains this through the relay and notifies the user that the device is already in the required state. Radio Frequency (RF) technology is used for wireless connectivity, which is a low cost, low power-consuming wireless networking technology, which can be directly interfaced to the encoders and microcontrollers. It has a maximum range of $100 \mathrm{ft}$ at $4800 \mathrm{bps}$ data rate.

\subsection{Automation through mobile}

Automation through mobile is a desired option when the user is outside the premises, where the appliances need to be controlled. Unlike other hand-held units, a mobile can be easily operated by the visually impaired and physically challenged [2]. DTMF technology is made use of in this technique. Like specific command words in automation through voice, specific control word numbers are fixed for specific appliances. The control module which is placed at the premises has a mobile circuitry and the user makes a call to that mobile circuitry. The mobile circuitry can either be a mobile phone to receive the call or a GSM modem for the same purpose. The mobile circuitry receives the call and this activates the control module. To provide security to the network so that nobody else makes a call to the mobile circuitry and misuses the facility, an authentication code is provided. Only when the user enters the authentication code correctly after making the call, then the control module functions. Once the correct authentication code is entered the user is authenticated. The user keys in respective control word numbers for a specific appliance to control it. The mobile circuitry in the control module within the premises receives this and sends to the microcontroller. The microcontroller after receiving the control word number acts accordingly and controls the relay. Thus the appliance connected to the relay is controlled.

DTMF signalling is used for telephone signalling over the line in the voice-frequency band to the call switching centre. The version of DTMF used for the telephone tone dialling is known by the trademark term Touch-Tone and is standardised by ITU-T Recommendation. Other multi-frequency systems are used for signalling internal to the telephone network. The DTMF keypad frequencies are shown in Table1. The DTMF keypad is laid out in a $4 \mathrm{X} 4$ matrix, with each row representing a low frequency, and each column representing a highfrequency. Pressing a single key will send a sinusoidal tone of the two frequencies. The original keypads had levers inside, so each button activated two contacts. The multiple tones are the reason for calling the system multi-frequency. These tones are then decoded by the switching canter to determine which key was pressed.

Table 1. DTMF keypad frequencies

\begin{tabular}{|c|c|c|c|c|}
\hline & $1209 \mathrm{~Hz}$ & $1336 \mathrm{~Hz}$ & $1477 \mathrm{~Hz}$ & $1633 \mathrm{~Hz}$ \\
\hline $697 \mathrm{~Hz}$ & 1 & 2 & 3 & $\mathrm{~A}$ \\
\hline $770 \mathrm{~Hz}$ & 4 & 5 & 6 & $B$ \\
\hline $852 \mathrm{~Hz}$ & 7 & 8 & 9 & $\mathrm{C}$ \\
\hline $941 \mathrm{~Hz}$ & $*$ & 0 & $\#$ & $D$ \\
\hline
\end{tabular}

The DTMF signals on the mobile internet are used as control signals. The DTMF chip CM8870 receives the call, decodes the DTMF tone from the keypad of the telephone into its respective $\mathrm{BCD}$ code after the system receives the call. This BCD code is decoded and the output is transmitted to the microcontroller connected to the relays, which in turn actuates the corresponding relay and hence the appliance. Here too a voice feedback is employed for the benefit of the user. The voice feedback is heard in the mobile itself soon after the control action has taken place. Thus the user can be assured that the control task has been accomplished. Here also if the user keys in the code to perform an action which has been already performed, the system gives back a message mentioning the same. The major advantage of this system is that the control can be overlapped, that is a device can be 
switched ON through voice and can be switched OFF through the mobile and vice versa.

\section{COMPONENTS DESCRIPTION} 4.1. HM2007-Voice Recognition Chip

HM2007 is a single chip CMOS voice recognition LSI circuit with the on-chip analog front end, voice analysis, voice recognition process and system control functions. Using the chip, a 40 isolated-word voice recognition system can be composed of external microphone, keyboard, 64k RAM and other additional circuitry. An intelligent recognition system can be built with the combination of microcontroller. The pin diagram of the HM2007 is shown in fig2.There are two operational modes in HM2007, they are,

- Manual mode

- $\quad$ CPU mode

\subsubsection{Manual mode}

The manual mode of operation is used in this project. In the manual mode, a keypad, an 8K-byte SRAM and other components may be connected to HM2007 to build a simple recognition system. When the power is on, HM2007 will start its initialization process. If WAIT pin is " $\mathrm{L}$ ", HM2007 will do the memory check to see whether the external $8 \mathrm{~K}$ byte SRAM is perfect or not. If WAIT pin is "H", "HM2007" will skip the memory check process. After the initial process is done, HM2007 will then move into recognition mode.

\subsubsection{Recognition Mode}

(a) WAIT PIN “ $H$ "

In this mode, the RDY [DIP PIN \#7] is set to low and HM2007 is ready to accept the voice input to be recognized. When the voice input is detected, the RDY will return to high and HM2007 begin its recognition process. It is recommended that user train the word pattern before beginning of the recognition operation, otherwise the result will be unpredictable. After the recognition process is completed the result will appear on the D-bus with the pin DEN [DIP PIN \#16] active.

Table 2. Content of the D-bus output in HM2007

\begin{tabular}{|llll|llll|l|}
\hline D7 & D6 & D5 & D4 & D7 & D6 & D5 & D4 & Description \\
\hline 0 & 0 & 0 & 0 & 0 & 0 & 0 & 0 & Power on \\
\hline \multicolumn{4}{|c|}{ A } & & \multicolumn{5}{|c|}{ B } & & Word AB \\
\hline 0 & 1 & 0 & 1 & 0 & 1 & 0 & 1 & Voice too long \\
\hline 0 & 1 & 1 & 0 & 0 & 1 & 1 & 0 & $\begin{array}{l}\text { Voice too } \\
\text { short }\end{array}$ \\
\hline 0 & 1 & 1 & 1 & 0 & 1 & 1 & 1 & Not match \\
\hline
\end{tabular}

Note 1: $\mathrm{A}$ is the binary code in the range 0 to 4 and $\mathrm{B}$ is the binary code in the range 0 to 9 .

Note 2: The maximum word length is $1.92 \mathrm{sec}$.

(b) WAIT PIN “ $L$ ”

In this mode, no voice input is accepted until WAIT [DIP PIN \#15] pin back to " $H$ " state. Two operations are included during this time, clearing a trained pattern and training a new pattern. To clear or train the voice pattern, one must select the word number to process first. The word is composed of two digits. The two digits are entered into HM2007 through keypad one digit a time. The word number is entered into HM2007through keypad one digit a time. When the word number is entered, press the function key to choose the operation function. If the function key CLR is pressed then the corresponding word pattern is cleared. HM2007 return to its recognition mode. If the function key TRN is pressed then HM2007 begins its tanning process. At the beginning of training process, if WAIT pin is "H", HM2007 sends a low level signal to RDY to indicate that HM2007 is ready to accept voice input. If WAIT pin is "L", no voice input is detected until WAIT pin back to "H". After available voice signal to RDY to indicate that HM2007 is ready for voice input to do HM2007, HM2007 returns to its recognition mode and send a low level signal to RDY to indicate that HM2007 returns to its recognition mode and send a low level signal to RDY to indicate that HM2007 is ready for voice input to do the recognition process. For example,

\section{TRN $\rightarrow$ Training the 24th pattern}

12 CLR $\rightarrow$ Clearing the 12th pattern

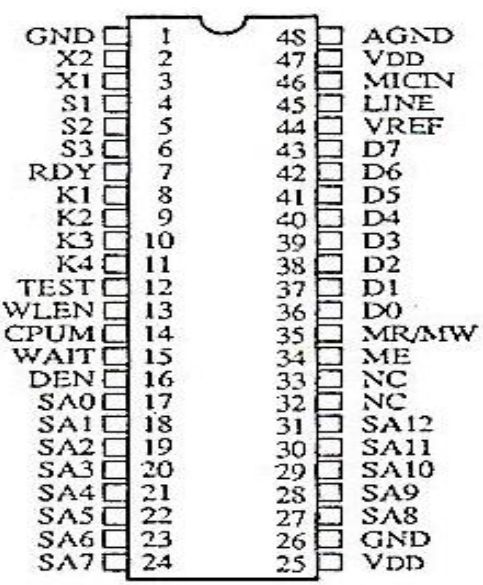

Fig 2. Pin diagram of HM2007P

\subsection{APR9600-Voice recording and playback}

The APR9600 device offers true single-chip voice recording, non-volatile storage and playback capability supporting both random and sequential access of multiple messages. Sample rates are user selectable allowing designers to customise their design for unique quality and storage time needs. Its proprietary analog/multilevel storage technology, where each memory cell can store up to 256 voltage levels enables the APR9600 device to reproduce voice signals in their natural form, eliminating the need for encoding and compression, which often introduce distortion. Figure3 shows interfacing the APR 9600.

\subsubsection{Message Management}

Playbook and record operations are managed by on chip circuitry. There are several available messaging modes, which determine message management style, message length and external parts count. The device supports three message management modes and they are,

- Random access mode with 2,4 or 8 fixed-duration messages.

- Tape mode with multiple variable-duration messages, provides two options:

$$
\circ \quad \text { Auto rewind }
$$




\section{Normal}

Random access mode supports 2, 4 or 8 message segments of fixed duration. As suggested, recording or playback can be made randomly in any of the selected messages. The length of each message segment is the total recording length available divided by the total number of segments enabled. Random access mode provides easy indexing to messages segments.

\subsection{CM8870-DTMF Receiver}

The CM8870 provides full DTMF receiver capability by integrating both the band-split decoder functions into a single 18-pin DIP. The filter section uses a switched capacitor technique for both high and low group filters and dial-tone rejection. It uses counting techniques for the detection and decoding of all 16 DTMF tone pairs into a 4-bit code. It also minimizes external component count by providing an on-chip differential input amplifier.

\subsection{HT12E-212 Series of Encoders}

HT12E is capable of encoding information which consists of $\mathrm{N}$ address bits and 12-N data bits. Each address/data bit can be set to one of the two logic states. The programmed address/data are transmitted together with the header bits via an RF medium. Interfacing the HT12E is shown in fig 4

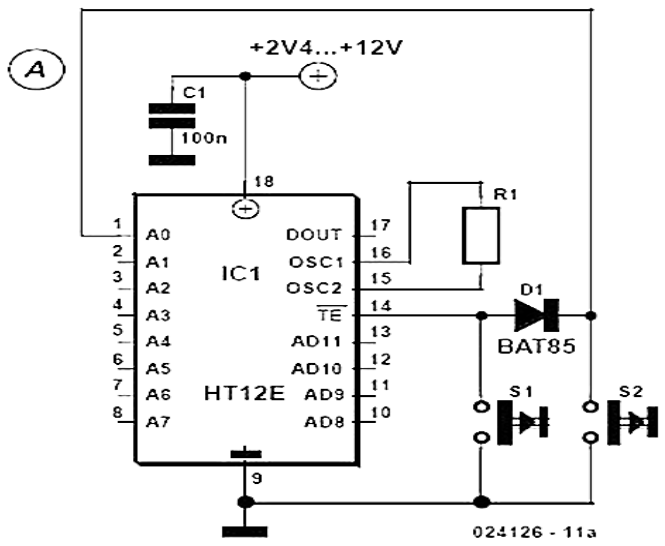

Fig 4. Interfacing the HT12E

\subsection{HT12D-212 Series of Decoders}

The HT12D decoders receive signal address and data from a programmed 212 series of encoders that are transmitted by a carrier using an RF. They compare the serial input data three times continuously with their local address. If no error or unmatched codes are found, the input data codes are decoded and then transferred to the output pins. Interfacing the HT12D is shown in fig 5.

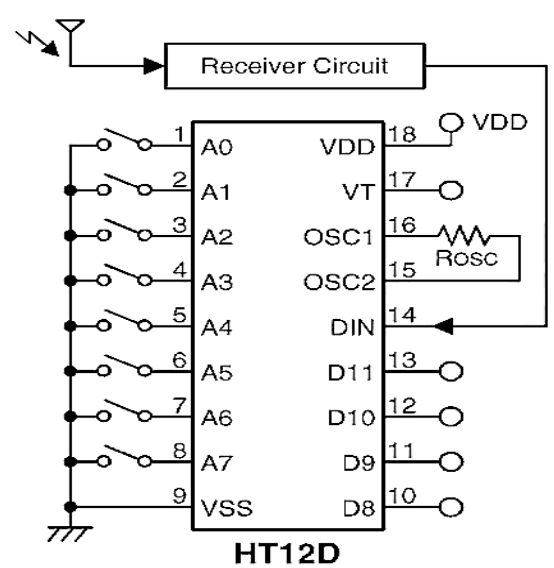

Fig 5. Interfacing the HT12D

\section{HARDWARE DESIGN}

The voice controlled and the mobile controlled home automation which interfaces HM2007 voice recognition chip and CM8870 DTMF decoder chip for respective control APR9600 chip is interfaced with the PIC microcontroller (M1) for voice feedback. Also the transmitter circuit is interfaced with the microcontroller to transmit the digital codes to the microcontroller (M2) connected to the relay circuitry and receive the codes from the relay circuitry. The circuit diagram of the proposed system's control unit and relay unit is shown in fig 6 and 7 .

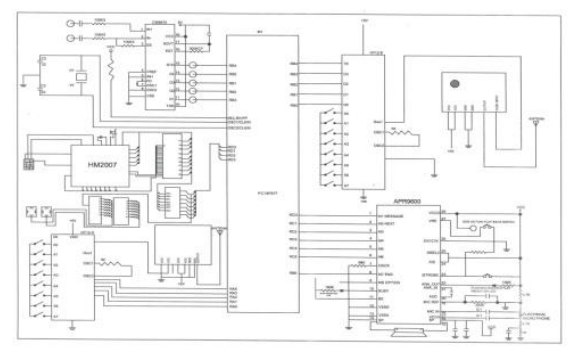

Fig 6. Circuit diagram of control unit

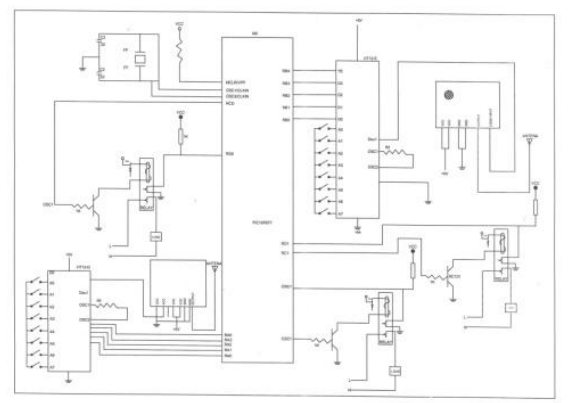

Fig 7. Circuit diagram of relay unit

\subsection{Voice Recognition}

An 8K SRAM is used since the HM2007 has built provision to interface it. First the HM2007 is put into training mode. In this mode, with the help of the keypad, predetermined control words for specific operations of specific device are stored in the SRAM. For example, the user might like to use the control word "Hall Light Off" to switch off the light in the hall. Now to store this in the SRAM a random location 21 is used. After 
keying the address 21 , the control word is spoken into the microphone, which gets stored in the SRAM. Similarly other control words for various other operations are stored in different address in the SRAM. Next the HM2007 is put into recognition mode, enabling the voice based control mechanism. Now when the user speaks a control word into the microphone, the HM2007 compares the input word with the previously stored control word in SRAM. If there is a successful match of the input word then a corresponding digital signal is given as the output, in which the microcontroller forwards to the Binary Coded Decimal (BCD) to 7-segment decoder and transmitted to the microcontroller (M2) connected to the relays.

\subsection{Voice Feedback Mechanism}

When the Microcontroller M2 enables the relay on successful recognition of the voice command input by the voice recognition circuit, it sends an acknowledgement code to M1 and a voice feedback is given to the user through an amplifier. This is achieved by interfacing the APR9600 with the microcontroller. Pre-determined voice feedbacks for each of the scenarios are stored in the APR9600 chip.

\subsection{Automation through Mobile}

When the user calls to the GSM in the mobile circuitry, the connection is established after a pre-determined number of rings. When the user keys in the pre-determined secret key, the mobile circuitry is activated and ready to receive the commands from the user mobile. The user keys in predetermined key combinations for respective tasks and respective DTMF signals are generated and sent to the mobile in the mobile circuitry. The CM8870 chip analyses the DTMF signals and decodes them into the respective $\mathrm{BCD}$ codes. The BCD codes are then encoded by the HT12-E encoders and transmitted from M1 to M2. On receipt of the codes by M2, it activates the corresponding relays. When the relay is activated a feedback message on receipt of the feedback signal is sent to M2, which transmits to M1. M1 plays the corresponding feedback message on receipt of the feedback signal.

\section{SOFTWAREDESIGN 6.1. Hi-tech C}

The language compiler used here is Hi-tech $\mathrm{C}$, which has an extremely good optimizer. It manages to produce tight, fast code, reasonably close to how it would be in assembly, which makes it an obvious choice for the PIC 16 family of microcontrollers. HI-TECH C Compiler for PIC10/12/16 MCUs supports a number of special features and extensions to the $\mathrm{C}$ language which are designed to ease the task of producing ROM-based applications. HI-TECH C has several features which relate directly to the PIC10/12/16 architectures and instruction sets.

\subsubsection{Stack}

The hardware stack on PIC devices is limited in depth and cannot be manipulated directly. It is only used for function return address and cannot be used for program data. The compiler implements a compiled stack for local data objects.

\subsubsection{Compiled Stack Operation}

Compiled stack operation is for information on how this is achieved. The user must ensure that the maximum stack depth is not exceeded otherwise code may fail. A call graph is provided by the code generator in the assembler list file. This will indicate the stack levels at each function call and can be used as a guide to stack depth. The code generator may also produce warnings if the maximum stack depth is exceeded.
Both of these are guides to stack usage. Optimizations and the use of interrupts can decrease or increase, respectively, the stack depth used by a program over that determined by the compiler.

\subsection{MPLAB IDE}

MPLAB IDE is a software program that runs on a pc to develop application for microchip microcontrollers. It is called an Integrated Development Environment, or IDE, because it provides a single integrated "environment" to develop code for embedded microcontrollers.A development system for embedded controller is a system of program running on a desktop PC to write, edit, debug and program code- the intelligence of embedded system applications into a microcontroller. MPLAB IDE runs on a PC and contains all the components needed to design and deploy embedded systems applications [12]. A typical task for developing an embedded controller application is to create the high level design. From the features and performance desired which PIC or dsPIC device is best suited to the application, and then design the associated hardware circuitry. After determining which peripherals and pins control the hardware, write the firmware-the software that will control the hardware aspects of the embedded applications. A language tool such as an assembler, which is directly translatable into machine code, or a compiler that allows a more natural language for creating programs should be used to write and edit codes. Assemblers and compilers make the code indestructible, allowing function labels to identify code routines with variables that have names associated with their use, and with constructs that helps to organize the code in a maintainable structure.

\subsubsection{MPLAB Features and Installation}

MPLAB IDE is a Windows OS based Integrated Development Environment for the PIC micro MCU families and the dsPIC Digital Signal Controllers. The MPLAB IDE provides the ability to

- Create and edit source code using built-in editor.

- Assemble, compile and link source code.

- Debug the executable logic by watching program flow with the built-in simulator or in real time.

- Make timing measurements with the simulator or emulator.

- View variables in watch windows.

- Program firmware into devices with device programmers.

\section{RESULTS AND DISCUSSION}

\subsection{Automation Through Voice}

Command words to control specific devices are first stored using the voice recognition kit with the help of a keypad in the RAM in the training mode. When the user speaks a command word during the recognition mode, the circuitry identifies whether the command word has been previously stored in the RAM or not. On successful recognition of the command word to control a specific device, the corresponding digital signal is sent to the microcontroller which control specific device through the relay circuitry. A voice feedback is given out for the benefit of the user as an acknowledgement. Light bulbs are used to simulate real time appliances.

\subsection{Training the Circuit}

In the circuitry memory location " 01 " in RAM is selected with the help of the keyboard and HM2007 is set to training mode. The command word "Light $1 \mathrm{ON}$ " is stored in that location by speaking into the microphone. Similarly the command word "Light 1 OFF" is stored in the memory 
location " 02 ". The feedback voice messages which are to be given out when the light is Switched ON or OFF like "Light 1 is switched ON" and "Light 1 is switched OFF" are stored in the APR9600 board by selecting the locations M1 and M2. Similarly in the memory locations " 03 " and " 04 " of the RAM, the command words "Light 2 ON" and "Light 2 OFF" are stored respectively. Also in the memory locations" 05 " and " 06 " of the RAM, the command words "Light 3 ON" and "Light 3 OFF" are stored respectively. The feedback message for light 2 and light 3 actions such as "Light 2 is switched on", "Light 2 is switched OFF", "Light 3 is Switched ON" and "Light 3 is switched OFF" are stored in the locations M3, M4, M5 and M6 of the APR9600 board respectively. The status message "Action already performed" is stored in location M7 of the APR 9600 board.

\subsection{Enabling a Device (Voice)}

HM2007 is set to recognition mode and the control word "Light 2 ON" is spoken into the microphone. Since there is a match for the command word in the memory, HM2007 sends the output word " 03 " to the microcontroller. The microcontroller on receipt of this activates the relay 2, connected to the light. Hence the light is switched ON and the feedback message "Light 2 is switched ON" is played.

\subsection{Disabling a Device (Voice)}

The command word "Light 2 OFF" spoken into the microphone in the recognition mode. The command word is recognized and HM2007 sends the output word " 04 " to the microcontroller. The microcontroller on the receipt of this input deactivates the relay 2, controlling the light. Thus the light is switched OFF and the feedback message "Light 2 is switched OFF" is played.

\subsection{Automation through Mobile}

The user calls to the mobile in the mobile circuitry and after predetermined number of rings the call is established. When the user inputs the secret key the mobile circuitry is activated. Then the user keys in the respective codes for enabling or disabling a device. The microcontroller, on receipt of these codes activates the relay and the voice feedback messages are played. The control words for the 3 light devices to be switched OFF are 02, 04 and 06 respectively.

\subsection{Enabling Device (Mobile)}

On the establishing the call, the secret key "777" is pressed, which activates the mobile circuitry. When the key pattern " 01 " is pressed, the decoder recognizes the control word and the corresponding digital code are transmitted to M2 from M1. The M2 receives the code and actuates the relay connected light and hence it is switched ON. M2 receives a feedback after the action and transmits it to M1, which plays the feedback message "Light 1 is switched ON".

\subsection{Disabling a Device (Mobile)}

On the establishing the call, the secret key "777" is pressed, which actuates the mobile circuitry. When the key "02" are pressed in succession, the decoder recognizes the control word and the corresponding digital code is sent to M2 from M1. M2 received the code and actuates the relay connected to light and hence it is switched OFF. M2 is receives a feedback after the action and transmits it to M1, which plays the feedback message "Light 1 switched OFF".

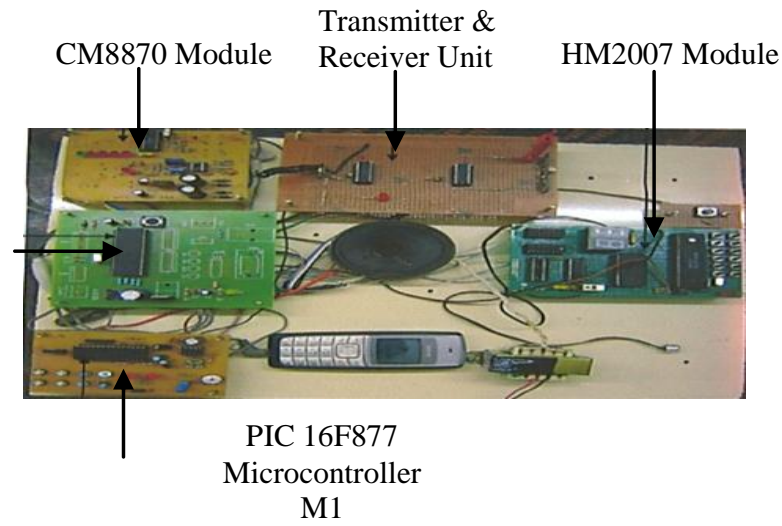

Fig.8 (a) Control unit

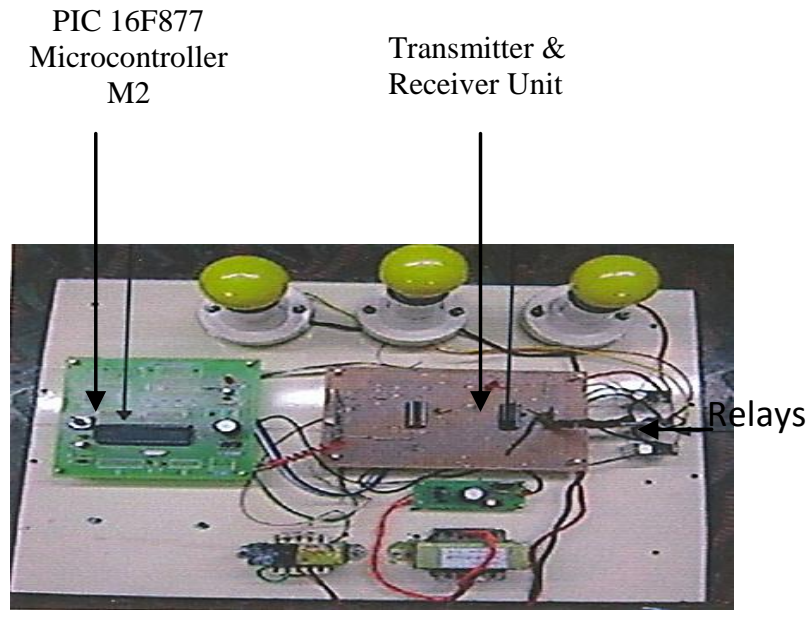

Fig.8 (b) Relay unit

\section{CONCLUSION}

Thus the automation through voice control and also through mobile control has been designed and implemented with voice feedback. A PIC16F877 microcontroller is used both at control unit (M1) and also at Relay unit (M2). A wireless connectivity between the microphone and the control circuitry was developed using Radio Frequency technology. DTMF technology has been used for the mobile control. Voice feedbacks which are very much essential for the acknowledgement of control action has been developed for the visually impaired and physically challenged people. The prototype of the proposed system is shown in fig $8(a)$ and 8(b).

\section{REFERENCES}

[1] M. Van Der Werf, X. Gui and W. L. Xu, "A mobilebased Home Automation System", $2^{\text {nd }}$ international Conference on Mobile Technology, Applications and Systems, Nov 2005, Page(s):5pp.

[2] Andreas Roendhal , J. Felix Hampe and GeotzBotterweek, "Mobile Home Automation -Merging Mobile Added Services and Home Automation Technologies", Sixth International Conference on the 
Management of Mobile Business (ICMB 2007), Page(s): 31-31.

[3] BarisYuksekkaya, A. AlperKayalar, M. BilehanTosum, M. KaanOzcan and Ali Ziya, "A GSM, Internet and Speech Controlled Wireless Interactive Home Automation System", IEEE Transactions on Consumer Electronics, Volume 52, Issue 3, August 2006, page(s):837-843.

[4] Renato Jorge CaleriaNunes, "Decentralized Supervision for Home Automation”, IEEE MELCON 2006, May 1619, Page(s):785-788.

[5] Ali ZiyaAlkar, "An Internet Based Wireless Home Automation System for Multifunctional Devices", IEEE Transaction on Consumer Electronics, Volume 51, Issue 4, November 2005, Page (5):1169-1174.

[6] KwangYeol Lee and Jae Weon Choi, "RemoteControlled Home Automation System via Bluetooth Home Network", SICE Annual Conference, August 4-6, 2003, Page (s):2824-2829.

[7] Do-HoonKim,Heung-GyoonRyu, “Obstacle Recognition system using Ultrasonic sensor and duplex RadioFrequency Camera for the visually impaired person”,ICACT 2011, Page(s):326-329.
[8] Rune Torbensen, "OHAS: Open Home Automation System, " IEEE International Symposium on Consumer Electronics, April 2008, Page(s):1-4.

[9] AzadehNazemi,Cesar Ortega-Sanchez,Iain Murray, "Digital Talking Book player for the visually impaired using FPGA",IEEE Computer society 2011, Page(s): 493-496.

[10] Zhi-Li and Guanglie ZHANG, "A Physical Activities Healthcare System Based on Wireless Sensing Techonolgy", $13^{\text {th }}$ IEEE International Conference on Embedded and Real-Time Computing System and Applications, 2002, Page(s): 369-376.

[11] Vasco Santos, Paulo Bartolomeu, Jose Fonseca and AlexandreMota, "B-Live-A Home Automation System for Disabled and Elderly People", IEEE International Conference on Intelligent Robots and Systems, 2005, Page(s) : 333-336.

[12] AtushiImadu, Tadokawai, Yogo Takada and Tomoki Tajiri, "Walking Guide Interface mechanism and Navigation system for the visually impaired", IEEE 2011, 